\title{
Monitorando a Degradação da Poliamida 11 (PA-11) via Espectroscopia na região do Infravermelho médio com Transformada de Fourier (FTIR)
}

\author{
Eloilson Domingos, Thieres M. C. Pereira, Eustáquio V. R. de Castro, Wanderson Romão, Geovane L. de Sena \\ Departamento de Química, UFES \\ Regina C. L. Guimarães \\ CENPES, PETROBRAS
}

\begin{abstract}
Resumo: O potencial da técnica de espectroscopia de infravermelho com transformada de Fourier e acessório de reflexão total atenuada (FTIR-ATR) foi avaliado para o monitoramento da degradação da poliamida 11 (PA-11) usada em dutos flexíveis. As amostras foram submetidas a envelhecimento em reatores com pressão controlada e atmosfera inerte. Os corpos de prova foram imersos em água deionizada (pH 7), nas temperaturas de 110,120 e $140{ }^{\circ} \mathrm{C}$ por um período de até 50 dias. A técnica recomendada para monitoramento da degradação da PA-11 é a viscosimetria, através de medidas da viscosidade inerente corrigida (VIC) e a análise termogravimétrica (TGA). O comportamento observado para a VIC e TGA durante o envelhecimento possibilitou a construção de modelos que correlacionam com a técnica de FTIR-ATR. A partir dos resultados de FTIR-ATR, a variação na intensidade da banda atribuída à fase amorfa, $1161 \mathrm{~cm}^{-1}$, possibilitou o monitoramento do envelhecimento quando associamos a técnica a modelos quimiométricos como o de regressão dos mínimos quadrados parciais, PLS. Portanto, a técnica FTIR-ATR pode ser uma nova alternativa no monitoramento da degradação hidrolítica da PA-11, eliminando assim o uso de solventes orgânicos tóxicos e reduzindo, consequentemente, o tempo de análise.
\end{abstract}

Palavras-chave: Poliamida 11, PA-11, degradação, espectroscopia na região do infravermelho, FTIR-ATR.

\section{Monitoring the Degradation of Polyamide 11 (PA-11) via Fourier Transform Infrared Spectroscopy (FTIR)}

\begin{abstract}
The potential of the infrared spectroscopy with the attenuated total reflection technique (FTIR-ATR) was evaluated to monitor degradation of polyamide 11, PA-11, applied in flexible pipes. The samples were subjected to aging on reactors with controlled pressure and atmosphere. The samples were immersed in deionized water, $\mathrm{pH} 7$, at temperatures of 110,120 and $140{ }^{\circ} \mathrm{C}$ over a period of up to 50 days. The typical technique recommended for monitoring PA-11 degradation is viscometry from inherent viscosity corrected (VIC) and thermogravimetric (TGA) measurements. TGA and VIC results allowed the use of chemometric models that can be related to FTIR-ATR spectra, with assignment of a band to the amorphous phase $\left(1161 \mathrm{~cm}^{-1}\right)$ of PA-11. The chemometric model generated from PLS data was satisfactory in predicting the aging of PA-11. Therefore, FTIR-ATR can be used as a new analytical methodology to monitor PA-11 degradation, thus eliminating the use of toxic organic solvents and reducing the time required for the analysis.
\end{abstract}

Keywords: Polyamide 11, PA-11, degradation, infrared spectroscopy, FTIR-ATR.

\section{Introdução}

Os dutos flexíveis são estruturas fundamentais para o transporte do petróleo entre o poço situado ao fundo do oceano e as plataformas flutuantes de produção. Eles são capazes de acompanhar os grandes deslocamentos deste sistema. São vantajosos em relação aos dutos rígidos de aço, pois são fabricados em longos comprimentos e armazenados em carretéis, facilitando o transporte e a instalação ${ }^{[1]}$. Essas linhas flexíveis são estruturas multicamadas formadas por aço e polímeros sobrepostos ou concêntricos, dispostos em um arranjo helicoidal que proporciona uma alta flexibilidade (Figura 1) ${ }^{[2]}$.

A quantidade de linhas flexíveis instaladas ao longo dos últimos anos em produções offshore de petróleo vem crescendo atualmente, devido a descobertas de novas reservas de petróleo (a reserva do pré-sal, por exemplo ${ }^{[3]}$ e como conseqüência, a avaliação da influência dos fatores ambientais sobre as propriedades físicoquímicas desses materiais vem ganhando importância ${ }^{[4]}$.

$\mathrm{Na}$ década de 70 , a poliamida 11 , PA-11, foi o material selecionado para ser utilizado como camada interna ou barreira de pressão de dutos flexíveis da indústria do petróleo ${ }^{[5]}$. A alta proporção de hidrocarbonetos em relação ao grupo amida $\left(-\left(\mathrm{CH}_{2}\right)_{10} \mathrm{CONH}-\right)$, fez com que a PA-11 apresentasse excelentes propriedades mecânicas (alta resistência à fadiga, baixo coeficiente de fricção) e boa resistência química, fazendo com que esse termoplástico semicristalino atue como material de barreira durante o transporte da mistura gás, água e óleo ${ }^{[6]}$.

A PA-11 é comercialmente preparada por uma reação de condensação do monômero ácido $\omega$-aminoundecanóico. Em tubos flexíveis a adição de um plastificante a estrutura polimérica tornouse necessário devido à modificação de algumas propriedades mecânicas da PA-11. A Tabela 1 mostra a variação dessas propriedades com e sem plastificante, onde o módulo de flexão e a tensão no ponto de escoamento são as propriedades mecânicas mais afetadas.

O plastificante comercialmente utilizado é o benzenobutilsulfonamida correspondendo a 12 wt. (\%) do material polimérico. O plastificante atua reduzindo o número de ligações de hidrogênio entre as moléculas da PA-11, interferindo diretamente em suas forças intermoleculares. Hoje, existe um 


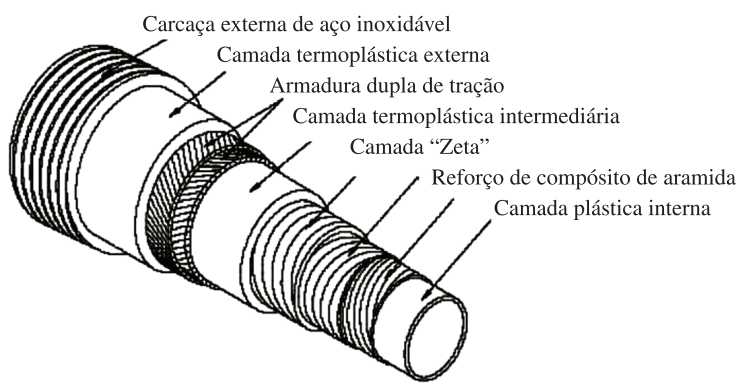

Figura 1. Duto flexível usado na exploração de petróleo. Figura adapatada a partir da literatura ${ }^{[1,2]}$.

Tabela 1. Propriedades mecânicas da PA-11 com e sem plastificante ${ }^{[6]}$.

\begin{tabular}{lccc}
\hline \multicolumn{1}{c}{$\begin{array}{c}\text { Propriedades } \\
\text { físico-químicas }\end{array}$} & Método & $\begin{array}{c}\text { PA-11 sem } \\
\text { plastificante }\end{array}$ & $\begin{array}{c}\text { PA-11 com } \\
\text { plastificante }\end{array}$ \\
\hline Ponto de Fusão $\left({ }^{\circ} \mathrm{C}\right)$ & ASTM D789 & $183-187$ & $178-184$ \\
Densidade $\left(\mathrm{g} \mathrm{cm}^{-3}\right)$ & ISO R 1183 D & 1,03 & 1,05 \\
$\begin{array}{l}\text { Tensão no ponto } \\
\text { de escoamento }\end{array}$ & ISO R 527 & 42 & 27 \\
Módulo de Flexão & ISO 178 & 1000 & 350 \\
\hline
\end{tabular}

especial interesse em se estudar o limite das propriedades físicoquímicas desse material necessárias para preservar a segurança das instalações offshore ${ }^{[6]}$.

A sobreposição de vários efeitos tem levado ao envelhecimento da PA-11 e a inevitável perda de suas propriedades físico-químicas. Os principais fatores que catalisam o processo de degradação são: água contida nos fluidos, temperatura, acidez $\left(\mathrm{CO}_{2}, \mathrm{H}_{2} \mathrm{~S}\right.$, ácidos orgânicos, ácidos inorgânicos), composição do óleo e a presença de outros produtos químicos utilizados no tratamento do óleo. Como conseqüência, algumas propriedades desse termoplástico podem ser alteradas como: perda de plastificante e, consequentemente, de elasticidade (veja na Tabela 1); variações em sua morfologia cristalina; e principalmente diminuição da massa molar devido à reação hidrolítica que acontece na presença de água ${ }^{[7]}$.

Sem dúvida, a água presente durante a produção de petróleo é o grande responsável pelo envelhecimento acelerado da PA-1 $11^{[6]}$. Ela provoca a hidrólise das ligações amídicas principalmente na matriz amorfa do polímero, catalisado pela temperatura e acidez, reduzindo diretamente a sua massa molar. A reação de hidrólise é reversível e é apresentada de forma simplificada pela Figura 2.

A técnica analítica normalmente empregada no monitoramento da degradação hidrolítica da PA-11 é a viscosimetria, a partir do monitoramento da viscosidade inerente. Entretanto, a metodologia analítica usada requer o uso de solventes tóxicos como o $m$-cresol e, consequentemente, volumes de resíduos orgânicos são gerados diariamente. Neste trabalho é proposto um método analítico alternativo para o monitoramento da degradação da PA-11: espectroscopia na região do infravermelho médio com transformada de Fourier e acessório de reflexão total atenuada, FTIR-ATR. A FTIR-ATR é uma técnica limpa e rápida que empregada juntamente com ferramentas quimiométricas (como o método de mínimos quadrados parciais, PLS) podem ser correlacionadas com medidas de viscosidade.

\section{Procedimento Experimental}

\section{Materiais e reagentes}

As amostras de PA-11 contendo 10-12\% do plastificante n-butilbenzenosulfonamida foram fornecidas pela Petrobrás S.A/Cenpes; elas foram cortadas com $13.0 \times 34.5 \times 5.5 \mathrm{~mm}$ de dimensão.

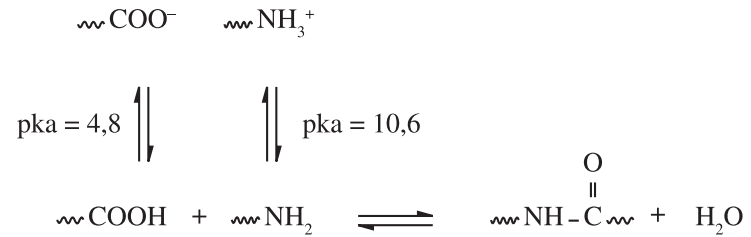

Figura 2. A reação global envolvendo o equilíbrio policondensação hidrólise $e^{[6,7]}$.

Para os ensaios de envelhecimento foi utilizado água deionizada $(\mathrm{pH} 7)$ de um sistema de purificação de água Permution e para as medidas de viscosidade, $m$-cresol (VETEC) foi usado como solvente.

\section{Ensaios de envelhecimento}

Os ensaios de envelhecimento foram realizados em água deionizada $(\mathrm{pH} 7)$ em reatores da marca Parr com um volume de 2 L. Para prevenir o envelhecimento oxidativo durante os ensaios, o sistema foi desaerado por um período de uma hora com uma vazão na linha de $2 \mathrm{~L} / \mathrm{min}$ com Argônio 5.0 ultra puro (99.999\%) ${ }^{[7]}$. Em seguida os reatores foram aquecidos e mantidos nas temperaturas 110,120 e $140 \pm 3{ }^{\circ} \mathrm{C}$. Os ensaios foram realizados durante 50 dias para as temperaturas de 110 e $120^{\circ} \mathrm{C}$; e 30 dias para a temperatura de $140{ }^{\circ} \mathrm{C}$. Em intervalos de tempo regulares, foram feitas aproximadamente 12 retiradas de corpos de prova para cada temperatura estudada. Em seguida, as amostras foram armazenadas em ambientes secos, escuros e frios $\left(\mathrm{T}<20^{\circ} \mathrm{C}\right)^{[6]}$.

\section{Caracterização}

\section{Viscosidade Inerente corrigida (VIC)}

As medidas de viscosidade inerente corrigida, VIC, seguiram o método ISO 307 (1994). Antes da realização das medidas de VIC foi necessário eliminar a umidade adsorvida na matriz polimérica. As amostras foram secadas utilizando uma estufa a vácuo $(<0,1 \mathrm{mbar})$, a uma temperatura de $80{ }^{\circ} \mathrm{C}$, durante um período de 12 horas. Posteriormente, uma concentração de $0,50 \pm 0,02$ g.dL ${ }^{-1}$ de PA-11 foi preparada usando $m$-cresol como solvente. As medidas de VIC foram realizadas utilizando um viscosímetro semi-automático D15KP-LAUDA a uma temperatura de 20,00 $\pm 0,01{ }^{\circ} \mathrm{C}$. O viscosímetro utilizado foi do tipo ubbelohde. Primeiramente, foi calculado a viscosidade inerente, VI, de acordo com a Equação 1.

$$
n_{\text {inh }}=\frac{\ln n_{r}}{c}=n_{\text {inh }}=\frac{\ln \left(t / t_{0}\right)}{c}
$$

onde $t_{0}=$ tempo de escoamento do solvente (segundos); $t=$ tempo de escoamento da solução (segundos); e $c=$ concentração $\left(\mathrm{g} \cdot \mathrm{dL}^{-1}\right)^{[6,7]}$.

Como a PA-11 não é um material comercialmente puro devido à presença do plastificante, foi preciso corrigir o valor da VI, a patir da Equação 2, fonecendo agora a VIC:

$$
V I C=V I \times\left(\frac{100}{100-\text { extraíveis }}\right)
$$

onde o percentual de extraíves corresponde ao wt. (\%) de aditivos ou impurezas.

De acordo com a API TECHNICAL REPORT $17 \mathrm{TR} 2^{[6]}$, as medidas de VIC devem obedecer uma repetibilidade de $3 \%$ e uma reprodutividade de $10 \%$. As análises de TGA foram realizadas a fim de se obter o percentual de extraiveis na PA-11. As curvas de TGA 
foram obtidas utilizando o TGA-DTA modelo SDTQ 600, marca TA. Aproximadamente $10 \mathrm{mg}$ da PA-11 foi submetida a uma taxa de aquecimento de $10{ }^{\circ} \mathrm{C} / \mathrm{min}$, sob atmosfera de nitrogênio, em um intervalo de temperatura de 25 a $210^{\circ} \mathrm{C}$.

\section{FTIR-ATR}

Os espectros de infravermelho médio foram obtidos em um espectrômetro modelo FTLA2000-102, do fabricante ABB BOMEM, usando como acessório uma célula horizontal de ATR (ZnSe, $45^{\circ}$ ) de $80 \mathrm{~mm}$ de comprimento, $10 \mathrm{~mm}$ de largura e $4 \mathrm{~mm}$ de espessura. $\mathrm{O}$ espectro registrado foi obtido como a média de 16 varreduras consecutivas, com resolução de $4 \mathrm{~cm}^{-1}$ e adquiridos em um intervalo de número de onda 4000 a $630 \mathrm{~cm}^{-1}$. Para a aquisição dos espectros foi utilizado o programa GRAMS/AI da Termo Galactic. Antes da obtenção de cada espectro, os corpos de prova sofreram um tratamento com lixas 120, 400, 600 e 1200 de granulometria com o objetivo de uniformizar a área superficial de trabalho.

\section{Quimiometria}

Inicialmente, foi aplicado um pré-tratamento (derivada primeira) na matriz de dados gerados a partir dos espectros de FTIR. Em seguida, os modelos de PLS foram desenvolvidos no ambiente MatLab R2009 e avaliados pela raiz quadrada da média quadrática dos erros de calibração (RMSECV).

\section{Resultados e Discussão}

Quando a degradação hidrolítica da PA-11 é catalisada pelo aumento da temperatura, $\left(\geq 110{ }^{\circ} \mathrm{C}\right)$, a liberação do plastificante benzeno-butilsulfonamida é acelerada para o meio aquoso ${ }^{[7]}$. Esse comportamento é confirmado pela Figura 3, a partir do monitoramento da porcentagem de plastificantes vs o tempo de envelhecimento obtido via medidas de TGA. Uma perda exponencial do plastificante é observado, sendo influenciada diretamente pelo aumento da temperatura. A rápida perda do plastificante é atribuída ao aumento da solubilidade em função da temperatura. Logo, a sua perda atinge um mínimo de $2 \mathrm{wt}$. (\%) na PA- 11 a $140{ }^{\circ} \mathrm{C}$ após 10 dias de envelhecimento. A temperatuas inferiores como 110 e $120{ }^{\circ} \mathrm{C}$, esse valor é relativamente maior sendo 2,5 wt. (\%) e 3 wt. (\%) após 30 e 19 dias de envelhecimento, respectivamente, Figura 3.

A VIC também foi monitorada em função do tempo de envelhecimento, Figura 4. Um comportamento exponencial dependente do aumento temperatura foi observado. A queda inicial da VIC se mostra mais acentuada em $140{ }^{\circ} \mathrm{C}$, indicando que a temperatura catalisa a reação de hidrólise, e consequentemente, a diminuição da massa molar da PA-11. A diminuição da VIC pode ser relacionada com as propriedades mecânicas. Por exemplo, a VIC limite permitida para uma aplicação segura da PA-11 nos risers, é 1,20 dL. $\mathrm{g}^{-1}$. Neste estudo, esse valor é atingido após 25,10 e 5 dias de envelhecimento em temperaturas de $110{ }^{\circ} \mathrm{C}, 120^{\circ} \mathrm{C}$ e $140{ }^{\circ} \mathrm{C}$. Em geral, as curvas obtidas a $120^{\circ} \mathrm{C} \mathrm{e} 140{ }^{\circ} \mathrm{C}$ atingiram um patamar de equilíbrio com valores menores que $1,20 \mathrm{dL} \cdot \mathrm{g}^{-1}$. Já a curva de $110{ }^{\circ} \mathrm{C}$ mostrou um patamar de equilíbrio acima de $1,20 \mathrm{dL} \cdot \mathrm{g}^{-1}$, Figura 4. Portanto, isso sugere que temperaturas acima de $110^{\circ} \mathrm{C}$ não são recomendadas para operações com a PA-11.

Para o desenvolvimento do novo método analítico a partir das medidas de FTIR-ATR, um espectro da PA-11 virgem foi adquirido, sendo mostrado na Figura 5. As principais bandas são enumeradas e identificadas na Tabela 2, onde ela descreve as atribuições das bandas encontradas.

Várias regiões do espectro de FTIR da PA-11 envelhecida foram avaliadas na tentativa de se correlacionar com os resultados de VIC.

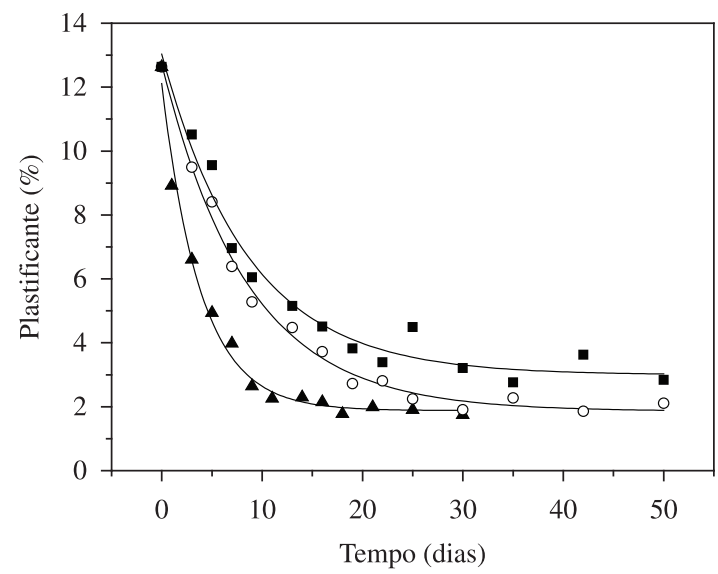

Figura 3. Perda do plastificante benzeno-butilsulfonamida $v s$ tempo de envelhecimento para temperaturas de $110^{\circ} \mathrm{C}(\boldsymbol{\square}), 120^{\circ} \mathrm{C}(\mathrm{\circ})$ e $140{ }^{\circ} \mathrm{C}(\boldsymbol{\Delta})$.

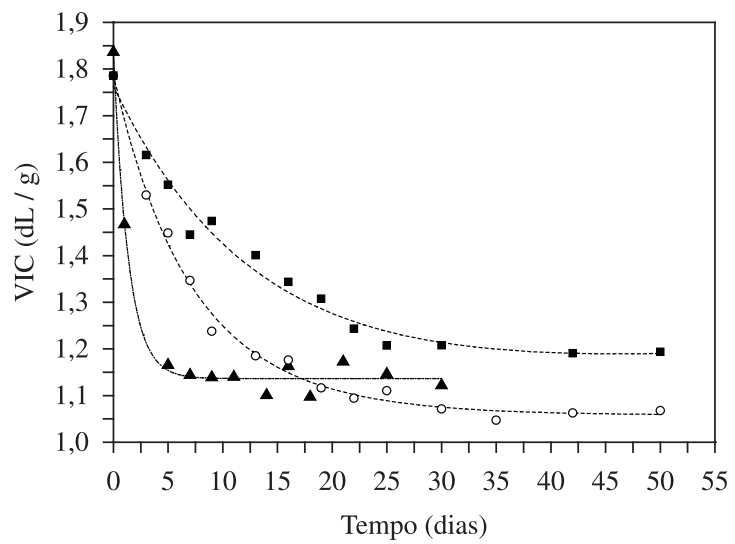

Figura 4. Viscosidade inerente corrigida das curvas $110^{\circ} \mathrm{C}(\boldsymbol{\square}), 120^{\circ} \mathrm{C}(\mathrm{O})$ e $140{ }^{\circ} \mathrm{C}(\boldsymbol{\Delta})$.

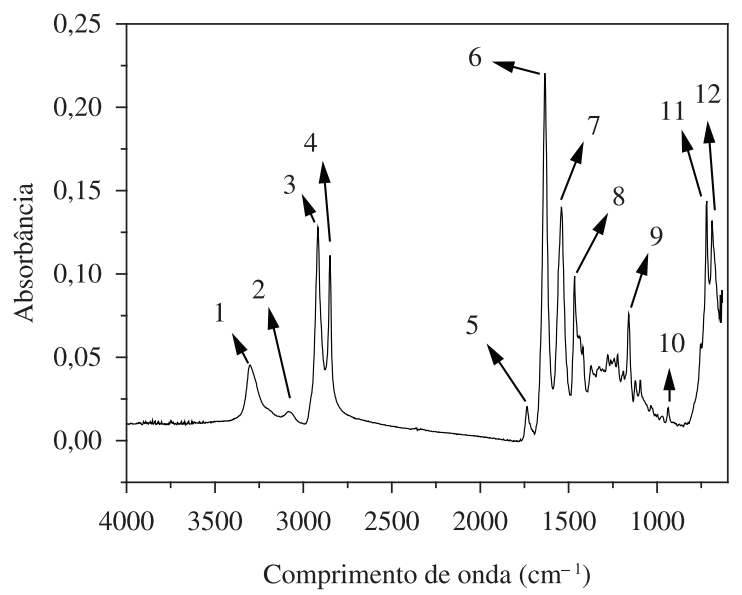

Figura 5. Espectro de FTIR da PA 11 nova

Os melhores resultados foram encontrados quando se monitora a variação da intensidade da banda de $1161 \mathrm{~cm}^{-1}$ em função do tempo de envelhecimento. A Figura 6 mostra a variação da intensidade (em absorbância) da banda $1161 \mathrm{~cm}^{-1} v s$ tempo para as 3 diferentes temperaturas estudadas $\left(110^{\circ} \mathrm{C}, 120^{\circ} \mathrm{C}\right.$, e $\left.140^{\circ} \mathrm{C}\right)$. A diminuição da intensidade dessa banda é diretamente atribuída a modificações na matriz polimérica referente a parte amorfa da PA-11. Romão et al. ${ }^{[7]}$ mostrou, por medidas de difração de raio $\mathrm{X}$, que o processo de degradação hidrolítica, ocorre preferencialmente na região amorfa PA-11. 
Estudos feitos por Svoboda et al. ${ }^{[8]}$ mostram que a cristalinidade da PA-11 pode ser acompanhada pela razão das intensidades 937 e $1161 \mathrm{~cm}^{-1}$, que correspondem as fases cristalinas e amorfas, respectivamente. A Figura 7 mostra a razão da intensidade dos

Tabela 2. Atribuição das bandas para a PA-11 virgem ${ }^{[8,9]}$.

\begin{tabular}{lll}
\hline *Banda & $\left(\mathbf{c m}^{-1}\right)$ & \multicolumn{1}{c}{ Atribuiçães } \\
\hline 1 & 3302 & Ligação N-H \\
2 & 3082 & ressonância de Fermi $(\delta \mathrm{NH}$ amida II $)$ \\
3 & 2918 & estiramento assimétrico de $\mathrm{CH}_{2}$ \\
4 & 2848 & estiramento simétrico de $\mathrm{CH}_{2}$ \\
5 & 1734 & ésteres e impurezas \\
6 & 1634 & Amida I (Estiramento C=O) \\
7 & 1541 & Amida II (grupo de -NH-CO-) \\
8 & 1466 & bending de C=O e N- vicinal $\mathrm{CH}_{2}$ \\
9 & 1161 & Interação entre deformação $(\delta) \mathrm{NH}$ e estiramento \\
& & (v) O=C-N \\
10 & 937 & Amida IV - $\delta(\mathrm{CONH})$ \\
11 & 721 & CH ${ }_{2}$ Rocking \\
12 & 688 & Amida $\mathrm{V}(\alpha)(\mathrm{CONH}$, deformação fora do plano $)$ \\
\hline
\end{tabular}

*Numerações referentes a Figura 5.
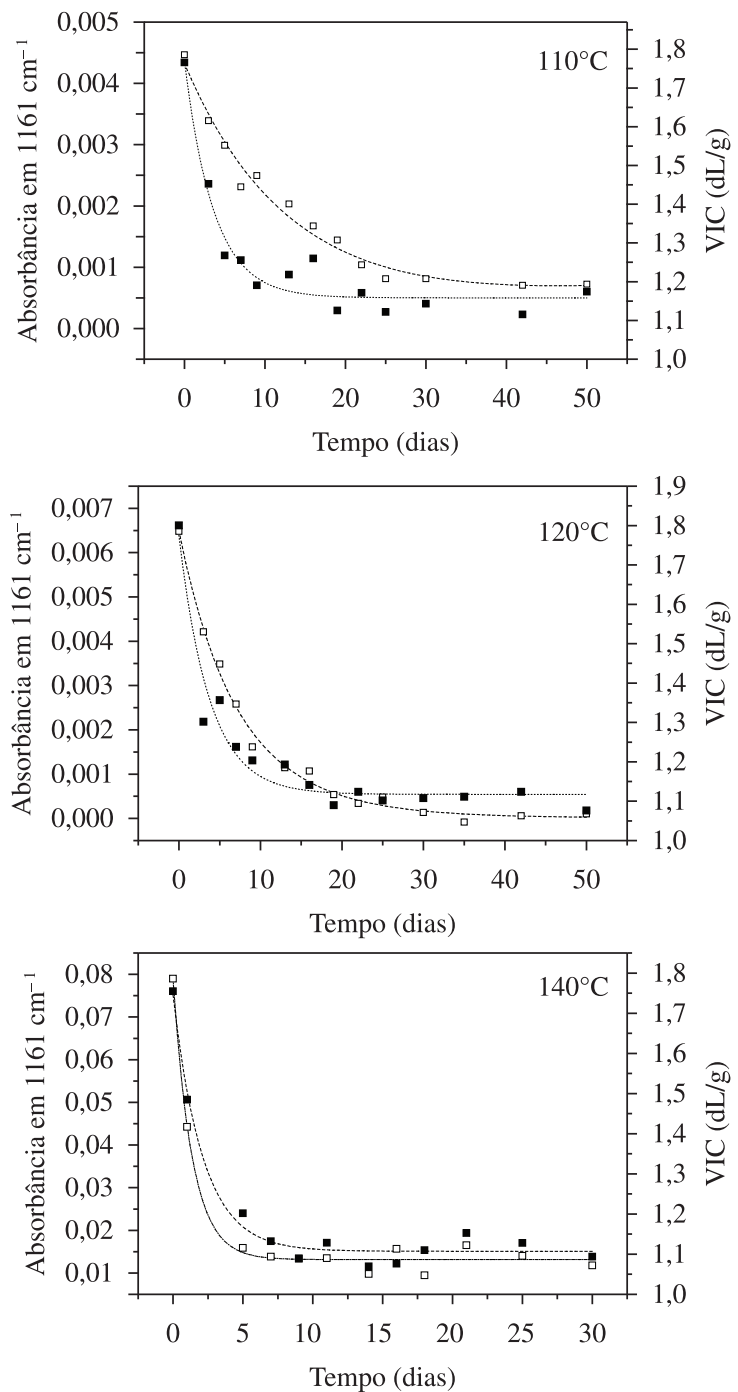

Figura 6. Correlação do número de onda de $1161 \mathrm{~cm}^{-1}$ via FTIR-ATR com os resultados de VIC $v s$ o tempo de envelhecimento nas temperaturas de 110 , 120 e $140{ }^{\circ} \mathrm{C}$. picos $937 \mathrm{~cm}^{-1} / 1161 \mathrm{~cm}^{-1}$ vs o tempo de envelhecimento. A razão obtida no intervalo de 0,6-0,7 corresponde a um aumento na cristalinidade em torno de 30 a $40 \%$, corroborando com resultados obtidos por técnicas clássicas como a calorimetria diferencial de varredura ${ }^{[10]}$. Esse aumento de cristalinidade (que também é influenciada pela temperatura, veja a intensidade da Figura 7) é explicado pela hidrolise sofrida pelo material ao longo do ensaio de envelhecimento, e consequente aumento das ligações de hidrogênio intermoleculares.

A aplicação da base de cálculo do iPLS seleciona as regiões espectrais que possuem um menor erro RMSECV. Com a divisão do espectro em 80 intervalos, foi determinado a melhor região de regressão para a determinação da VIC, como sendo entre $1143 \mathrm{~cm}^{-1}$ a $1184 \mathrm{~cm}^{-1}$, confirmando assim, que o pico em $1161 \mathrm{~cm}^{-1}$ está altamente correlacionado com o envelhecimento. A matriz de dados foi retirado a partir da primeira derivada dos espectros de FTIRATR das amostras de PA-11, nas curvas de envelhecimento a 110, 120 e $140{ }^{\circ} \mathrm{C}$. Um total de 37 amostras foram usadas. Dois grupos distintos foram avaliados: 26 amostras $(70 \%)$ como conjunto de calibração e $11(30 \%)$ como conjunto de previsão do modelo. A Figura 8 apresenta a relação entre os valores de VIC previstas pela técnica de FTIR-ATR e os resultados de VIC medidos a partir das medidas de viscosidade. Valores de regressão de 0,8185 e 0,6579 para calibração e previsão foram obtidos.

Este modelo apresentou RMSECV de 5,21\% a partir de 4 variáveis latentes e $97,0 \%$ da variabilidade dos dados obtidos
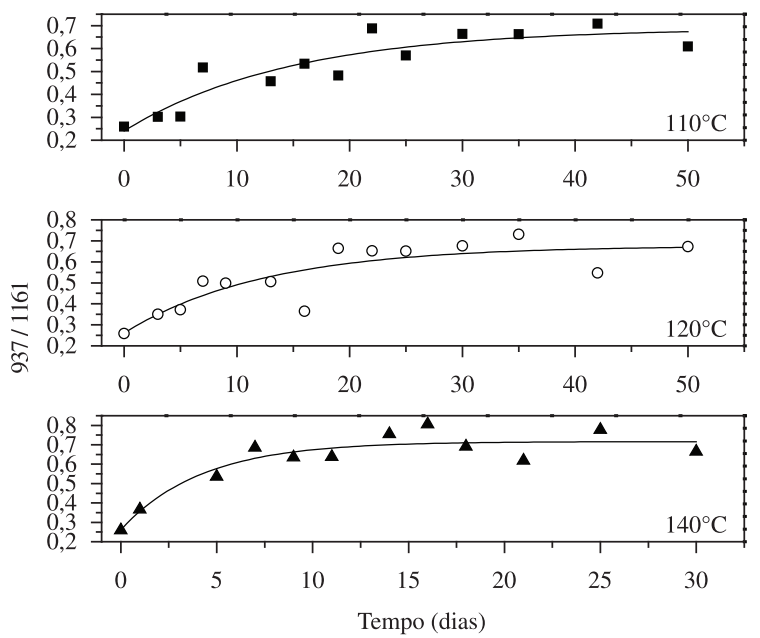

Figura 7. Razão da intensidade das bandas de número de onda de $937 / 1161 \mathrm{~cm}^{-1}$ em função do tempo de envelhecimento para as temperaturas de 110,120 e $140{ }^{\circ} \mathrm{C}$.

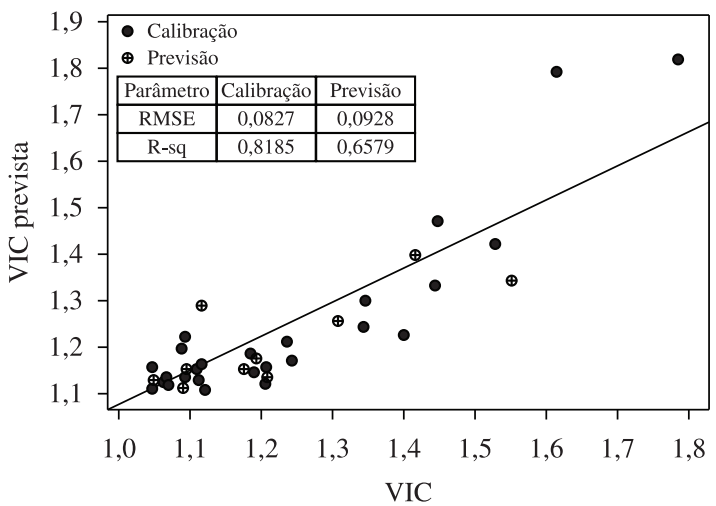

Figura 8. Gráfico de VIC medida versus VIC prevista pelo PLS. 


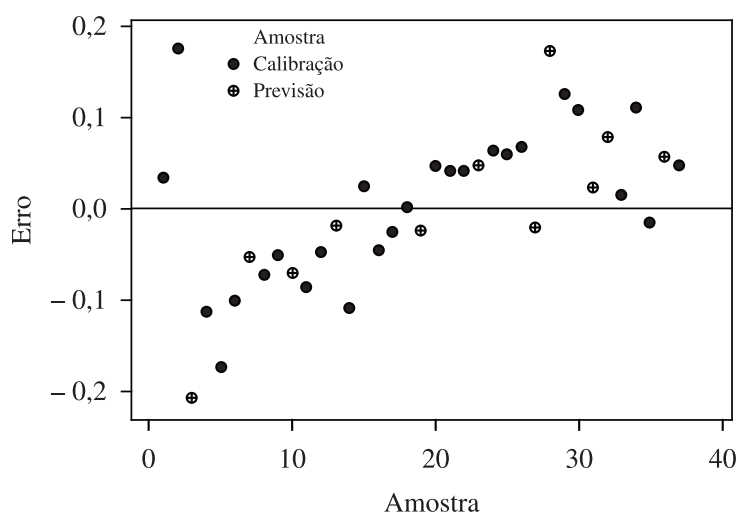

Figura 9. Gráfico de erros gerado na rotina de cálculo do PLS.

pelo PLS. A Figura 9 apresenta o intervalo de erros para cada amostra, sendo favorável para a previsão da VIC utilizando a técnica FTIR-ATR.

\section{Conclusão}

Os ensaios de degradação foram eficientes para a construção das curvas de envelhecimento. As medidas de VIC e wt. (\%) de extraíveis apresentaram uma queda exponencial com o tempo de envelhecimento e são catalisados pelo aumento da temperatura. A variação da VIC pode ser relacionada diretamente com a diminuição da massa molar da PA-11. Já a variação na wt. (\%) de extraíveis informa a diminuição da quantidade de aditivo na matriz polimérica durante o processo de degradação hidrolítica.

Os espectros gerados a partir da técnica de FTIR-ATR são de fácil obtenção, podendo monitorar as modificações sofridas a partir do processo de degradação hidrolítica. Entre as bandas encontradas no espectro, destacam-se as absorções na região de 937 e $1161 \mathrm{~cm}^{-1}$. Elas são capazes de acompanhar a degradação hidrolítica sofrida pelo material quanto as suas mudanças morfológicas.

Os modelos gerados pelos métodos de PLS foram satisfatórios na previsão do envelhecimento da PA-11, a partir da determinação da VIC. O coeficiente de correlação na construção do modelo a partir da técnica FTIR-ATR foi de 0,8185 . Entretanto, esse resultado pode ser melhorado, desde que a matriz de dados seja reformulada. Uma excelente alternativa seria o uso de amostras de PA-11 envelhecidas no campo de exploração de petróleo offshore, ou seja, o uso de amostras submetidas a condições "reais" de trabalho.

\section{Agradecimentos}

Os autores agradecem à CAPES, CNPq, FAPES e Petrobras por auxílio à pesquisa.

\section{Referências Bibliográficas}

1. Souza, A. P. F. - "Colapso de dutos flexíveis sob pressão externa", Dissertação de mestrado, Universidade Federal do Rio de Janeiro, Brasil (2002).

2. Costa, C. H. O. - "Correlação analítico - experimental de risers flexíveis submetidos a cargas radiais", Dissertação de Mestrado, Universidade Federal do Rio de Janeiro, Brasil (2006).

3. Botto, A.; Banti, C. \& O'sullivan, E. - "Managing ageing flexible pipe assets", in: Proceedings of the International Conference on Offshore Mechanics and Artic Engineering, Estoril, Portugal (2008).

4. Bispo, C. E. - "Efeito do envelhecimento da poliamida 11 em petróleo sobre as propriedades térmicas e mecânicas", Dissertação de Mestrado, Universidade Federal do Rio de Janeiro, Brasil (2008).

5. Olabisi, O. - "Handbook of Thermoplastics", v.41, Marcel Dekker, New York (1997).

6. API Technical Report 17TR2. - "The ageing of PA-11 in flexible pipes", American Petroleum Institute, Washington D.C. (2003).

7. Romão, W.; De Castro, E. V. R.; Silva Filho, E. A.; Guimaraes, R. C. L.; Silva, A. L. N.; Teixeira, S. C. S.; De Paoli, M. A. \& Sena, G. L. - J. Appl. Polym. Sci., 114, p.1777 (2009). http://dx.doi.org/10.1002/ app.30793

8. Svoboda, M.; Schneider, B. \& Stokr, J. - Collect. Czech. Chem. Commun., 56, p.1461 (1990).

9. Silverstein, R. M.; Bassler, G. C. \& Morrill, T. C. - "Spectrometric identification of organic compounds", John Wiley \& Sons, New York (1991).

10. Domingos, E. - "Utilização de Técnicas Espectroscópicas e Análise Térmica no Monitoramento da Degradação da Poliamida 11 Utilizada em Dutos Flexíveis", Dissertação de Mestrado, Universidade Federal do Espírito Santo, Brasil (2011).

Enviado: 01/11/11

Reenviado: 28/12/11

Aceito: 08/02/12 